\title{
Fraction of Inspired Oxygen as a Predictor of CPAP Failure in Preterm Infants with Respiratory Distress Syndrome: A Prospective Multicenter Study
}

\author{
Ewa Gulczyńska ${ }^{a}$ Tomasz Szczapa $^{\text {b }}$ Roman Hożejowski ${ }^{c}$ \\ Maria Katarzyna Borszewska-Kornacka ${ }^{d}$ Magdalena Rutkowska ${ }^{e}$ \\ a Department of Neonatology, Polish Mother's Memorial Hospital Research Institute, Lodz, Poland; \\ ${ }^{b}$ Department of Neonatology, Poznan University of Medical Sciences, Poznan, Poland; ${ }^{c}$ Medical Department, \\ Chiesi Poland, Warsaw, Poland; ${ }^{d}$ Neonatal and Intensive Care Department, Medical University of Warsaw, \\ Warsaw, Poland; ${ }^{\text {e}}$ Clinic of Neonatology and Intensive Care, Institute of Mother and Child, Warsaw, Poland
}

\section{Keywords}

Preterm infant . Continuous positive airway pressure . Respiratory distress syndrome - Mechanical ventilation . Fraction of inspired oxygen - Treatment failure

\section{Abstract \\ Introduction: There are limited data available regarding the fraction of inspired oxygen $\left(\mathrm{FiO}_{2}\right)$ predictive of the failure of continuous positive airway pressure (CPAP) in preterm in- fants with respiratory distress syndrome (RDS). Therefore, we investigated factors predictive of CPAP failure in the first $72 \mathrm{~h}$ of life, with special attention to the prognostic role of $\mathrm{FiO}_{2}$. Methods: This multicenter, prospective study enrolled infants $<30$ weeks gestation in whom CPAP was initiated within the first 15 min after birth. In the univariate and mul- tivariate logistic regression models, demographic, perinatal, and respiratory parameters were analyzed. The $\mathrm{FiO}_{2}$ thresh- old was determined with ROC curve analysis. Results: Of 389 recruited newborns, CPAP failure occurred in 108 infants (27.8\%). In the univariate model, each gestational week re- duced the odds of CPAP failure by $19 \%$, and each $100 \mathrm{~g}$ of birth weight reduced the odds by $16 \%$ (both $p<0.05$ ). The}

risk was increased by 4.2 and $7.5 \%$ for each 0.01 increase in $\mathrm{FiO}_{2}$ in the first and second hours of life, respectively. In the final multivariate model, birth weight and $\mathrm{FiO}_{2}$ in the second hour of life were the predictive measures. The prognostic threshold was $\mathrm{FiO}_{2}=0.29$ in the second hour of life (AUC 0.7; $p<0.0001$ ), with a sensitivity of $73 \%$ and a specificity of $57 \%$. CPAP failure implied a more than 20-fold higher risk of death and pneumothorax and a 2- to 5 -fold higher risk of typical complications of prematurity, including bronchopulmonary dysplasia and severe intraventricular hemorrhage. Conclusion: $\mathrm{FiO}_{2}$ in the second hour of life is a significant predictor of CPAP failure. The threshold of 0.29 best discriminates the CPAP outcome. Nonresponders to CPAP have a remarkably higher incidence of complications and a higher mortality rate.

(c) 2019 The Author(s)

Published by S. Karger AG, Basel

\section{Introduction}

The treatment of neonatal respiratory distress syndrome (RDS) with nasal continuous positive airway pressure (CPAP) is now widespread in preterm newborns

\begin{tabular}{ll}
\hline KARGER & $\begin{array}{l}\text { Karger } \\
\text { Published by S. Karger AG, Basel }\end{array}$ \\
E-Mail karger@karger.com & $\begin{array}{l}\text { This article is licensed under the Creative Commons Attribution- } \\
\text { NonCommercial-NoDerivatives 4.0 International License (CC BY- } \\
\text { NC-ND) (http://www.karger.com/Services/OpenAccessLicense). } \\
\text { Usage and distribution for commercial purposes as well as any dis- } \\
\text { tribution of modified material requires written permission. }\end{array}$
\end{tabular}

Prof. Ewa Gulczyńska

Department of Neonatology, Polish Mother's Memorial Hospital Research Institute ul. Rzgowska 281/289

PL-93-338 Lodz (Poland)

E-Mail ewagulcz@wp.pl 
who do not require intubation during post-birth stabilization $[1,2]$. According to the 2016 European Guidelines on the management of RDS, early nasal CPAP is recommended as the first-choice treatment in infants $<30$ weeks' gestation, who are at risk of RDS, but do not require mechanical ventilation (MV) [3]. The key clinical benefit of the early initiation of CPAP is the potential avoidance of invasive ventilation with all the related sequelae [4]. However, the efficacy of early CPAP may vary, and the success rate largely depends on the gestational age $[5,6]$. Of note, infants who fail CPAP are at increased risk of death, pneumothorax, and bronchopulmonary dysplasia (BPD), among other morbidities $[5,6]$.

Surfactant deficiency has been suggested to be the main cause of CPAP failure; therefore, from a clinical perspective, it is vital to identify surfactant-deficient infants as soon as possible and to introduce early rescue surfactant therapy. Several factors predictive of treatment failure have been proposed in the literature, of which the maximum fraction of inspired oxygen $\left(\mathrm{FiO}_{2}\right)$ in the first hours of life was most often reported [5, 6, 7-11]. Available data regarding the specific level of $\mathrm{FiO}_{2}$, which is predictive of CPAP failure, are still limited. Many experts pose the following questions: "which newborns have the highest risk of CPAP failure?" and "when should the decision on surfactant administration be made?"; thus, we conducted a multicenter, prospective trial to assess relevant prognostic factors and, most importantly, to define the $\mathrm{FiO}_{2}$ threshold indicative of likely CPAP failure.

\section{Patients and Methods}

This prospective, multicenter, cohort study was conducted from October 2016 to January 2018 in 29 tertiary referral neonatal intensive care units. The study protocol was approved by the $\mathrm{Hu}$ man Ethics Committee of Warsaw Medical University in accordance with the principal investigator's affiliation.

The enrollment criteria were: (1) preterm infants at risk of RDS, not requiring $\mathrm{MV}$ in the delivery room, (2) gestational age less than 30 weeks, and (3) CPAP initiated within 15 min of birth. The presence of clinically significant congenital defects was an exclusion criterion.

The incidence of CPAP failure was defined as the percentage of infants requiring invasive ventilation within the first $72 \mathrm{~h}$ of life. All study sites used the following criteria for invasive ventilation as established by the guidelines of the Polish Neonatal Society [12]: apnea not responding to stimulation, excessive breathing effort despite noninvasive respiratory support, hypercarbia $>55 \mathrm{~mm} \mathrm{Hg}$, and low blood oxygen tension $\left(\mathrm{PaO}_{2}<50 \mathrm{~mm} \mathrm{Hg}\right.$ or $\mathrm{SpO}_{2}<87 \%$ with $\mathrm{FiO}_{2}>0.6$ ).

If surfactant was required, centers with insufficient experience in less invasive surfactant administration (LISA) could use the INSURE technique (intubation-surfactant-extubation). INSURE did not determine failure of CPAP provided that the infant could be extubated within $30 \mathrm{~min}$ at the latest. The criteria for surfactant administration were $\mathrm{FiO}_{2}>0.3$ in infants $\leq 26$ weeks and $>0.4$ in infants $>26$ weeks. $\mathrm{FiO}_{2}$ in the first hours of life was defined as the highest oxygen concentration delivered to maintain a target saturation range of $90-94 \%$, as recommended by the European RDS guidelines 2016 .

To assess factors predictive of CPAP failure, we used both univariate and multivariate logistic regression analysis, in which demographic, perinatal, and respiratory parameters were independent variables. In multivariate analysis, we used a stepwise selection process, starting with the baseline model encompassing all candidate variables. The final model was developed using the Akaike information criterion (AIC) by subsequent dropping variables whose loss resulted in the most statistically insignificant deterioration in the model fit. For all predictive factors, odds ratios (OR) were computed with the corresponding $p$ values and $95 \%$ confidence intervals. To control for the impact of a multicentric design, two models, with and without a "center factor," were built. These were later compared with the AIC and the likelihood ratio test (LRT).

To analyze the impact of the $\mathrm{FiO}_{2}$ level in the first hours of life on the later occurrence of CPAP failure, we employed ROC (receiver operating characteristic) curve analysis. The test's ability to correctly classify patients as those who will develop CPAP failure or not is represented by the area under the ROC curve (AUC). The AUC can be in the range of $0.5-1.0$, wherein an AUC of 0.5 indicates that the classification model is of no value (random guessing), and a value of 1.0 designates perfect diagnostic accuracy. In turn, the cutoff point of the ROC curve designates the particular $\mathrm{FiO}_{2}$ level that gives the most true and the least false indications of CPAP failure, and therefore best predicts the treatment effect.

Additionally, clinical outcomes between the CPAP failure and CPAP success groups were compared using Pearson's $\chi^{2}$ test or Fisher's exact test. The outcomes encompassed the occurrence of death, respiratory complications including BPD, and other complications of prematurity: intraventricular hemorrhage (IVH), patent ductus arteriosus (PDA), periventricular leukomalacia, retinopathy of prematurity, and necrotizing enterocolitis.

BPD was diagnosed at 36 weeks gestational age or at hospital discharge, whichever occurred first, and the diagnostic criteria were the use of oxygen for at least 28 days and sustained oxygen dependency with $\mathrm{FiO}_{2}>0.30$ (severe $\mathrm{BPD}$ ), $\mathrm{FiO}_{2}<0.30$ (moderate BPD), or breathing room air (mild BPD). IVH was diagnosed using transfontanelle ultrasonography performed according to the approved standards and was further classified using Papile's grading system.

Statistical analysis was performed with R statistical software (version 3.2, Foundation for Statistical Computing, Vienna, Austria). For all analyses, two-sided $p$ values $<0.05$ were considered significant.

\section{Results}

\section{Participants}

A total of 394 infants at less than 30 weeks gestation were included in the study. The analysis excluded data from 5 neonates who were transferred to another unit/ hospital on the first day of life, were lost to follow-up 
Table 1. Patient characteristics stratified by CPAP outcome

\begin{tabular}{|c|c|c|c|c|}
\hline & All $(n=389)$ & CPAP failure $(n=108)$ & CPAP success $(n=281)$ & $p$ value $^{\mathrm{a}}$ \\
\hline \multicolumn{5}{|l|}{ Perinatal factors } \\
\hline Cesarean delivery & $325(83.5)$ & $95(88.0)$ & $230(81.9)$ & 0.170 \\
\hline Multiple birth & $86(22.1)$ & $24(22.2)$ & $62(22.1)$ & 1.000 \\
\hline Antenatal steroids & $349(89.7)$ & $100(92.6)$ & $249(89.2)$ & 0.446 \\
\hline \multicolumn{5}{|l|}{ Neonatal factors } \\
\hline Male gender & $194(49.9)$ & $55(50.9)$ & $139(49.6)$ & 0.909 \\
\hline 5-min Apgar score & $8[7-8]$ & $7[6-8]$ & $8[7-8]$ & 0.049 \\
\hline $\mathrm{FiO}_{2}-1$ st h of life & $0.35(0.12)$ & $0.4(0.17)$ & $0.33(0.1)$ & $<0.001$ \\
\hline $\mathrm{FiO}_{2}-2 \mathrm{nd} \mathrm{h}$ of life & $0.31(0.11)$ & $0.37(0.16)$ & $0.29(0.08)$ & $<0.001$ \\
\hline Exogenous surfactant & $236(60.7)$ & $102(94.4)$ & $132(47.0)$ & $<0.001$ \\
\hline
\end{tabular}

Data are presented as $n(\%)$, mean (SD), or median [IQR]. Significant $p$ values are indicated in bold. CPAP, continuous positive airway pressure; $\mathrm{FiO}_{2}$, fraction of inspired oxygen.

${ }^{a}$ CPAP failure vs. CPAP success.

$(n=4)$, or did not provide sufficient data $(n=1)$. Thus, the final analysis was performed with 389 infants.

This study population included almost identical numbers of boys and girls, with a mean gestational age of 28.2 weeks ( $\pm 1.2 \mathrm{SD})$. The mean birth weight was $1,120 \mathrm{~g}$, and half of the infants weighed between 940 and 1,300 g. The median Apgar score was 8 points (interquartile range, IQR, 7-8). A total of 325 (83.5\%) infants were delivered by cesarean section.

Of the 127 infants successfully treated with surfactant, LISA was used in 31 , and the INSURE technique was used in 96 (Fig. 1). The age at surfactant treatment was not significantly different between the two techniques (a median of $1.4 \mathrm{~h}$ for INSURE vs. $1.3 \mathrm{~h}$ for LISA; $p=0.205$ ). The median time to extubation following INSURE was $5 \mathrm{~min}$ (IQR 2-8).

Those infants who failed CPAP were of lower gestational age, birth weight, and Apgar scores at $5 \mathrm{~min}$, had higher oxygen requirements during the first hours after birth, and more often required exogenous surfactant. Detailed clinical characteristics are provided in Table 1.

\section{CPAP Failure and its Predictors}

Overall, CPAP failure occurred in 108 infants (27.8\%; Fig. 1), showing variable frequency depending on gestational age (failure rate from a high of $50 \%$ at $23-24$ weeks gestation to a low of $22.7 \%$ at 29 weeks gestation).
In the vast majority of infants, more than one criterion decisive for MV was reported. Excessive breathing effort had 85 indications (78.7\%), low blood oxygen tension had $60(55.6 \%)$, hypercarbia had $26(24.1 \%)$, and apnea had $25(23.1 \%)$.

In the univariate regression model explaining CPAP failure, the following factors were significant: gestational age, birth weight, and $\mathrm{FiO}_{2}$ level, both in the first and second hour of life. An increase in gestational age and birth weight reduced the odds for CPAP failure by $19 \%$ per gestational week and by $16 \%$ per $100 \mathrm{~g}$ of birth weight, respectively. In contrast, higher $\mathrm{FiO}_{2}$ levels both in the first and second hour of life increased the odds for CPAP failure by 4.2 and $7.5 \%$, respectively, per each percentage point of oxygen concentration (Table 2).

In the final multivariate regression model, birth weight and $\mathrm{FiO}_{2}$ in the second hour of life were the predictive measures. Multiple treatment centers had no impact on the factors of interest (final CPAP predictors), and the model that did not include a center factor had significantly better goodness of fit (LRT $p=0.017$ ) and parsimony (AIC 404.3 vs. 414.3).

In ROC analysis, $\mathrm{FiO}_{2}$ in the second hour of life was a statistically significant predictor of CPAP failure, with an AUC of 0.7 and 95\% CI 0.62-0.74 ( $p<0.0001)$, showing a prognostic cutoff point at $\mathrm{FiO}_{2}=0.29$, with a sensitivity of $73 \%$ and a specificity of $57 \%$ (Fig. 2). 
Fig. 1. Flow chart of included patients. MV, mechanical ventilation; LISA, less invasive surfactant administration; INSURE, intubation, surfactant, extubation. * Of 61 ventilated infants, $55(90 \%)$ received surfactant. ** Of 27 ventilated infants, 6 (22\%) received surfactant. \# Most patients had more than 1 indication for MV.

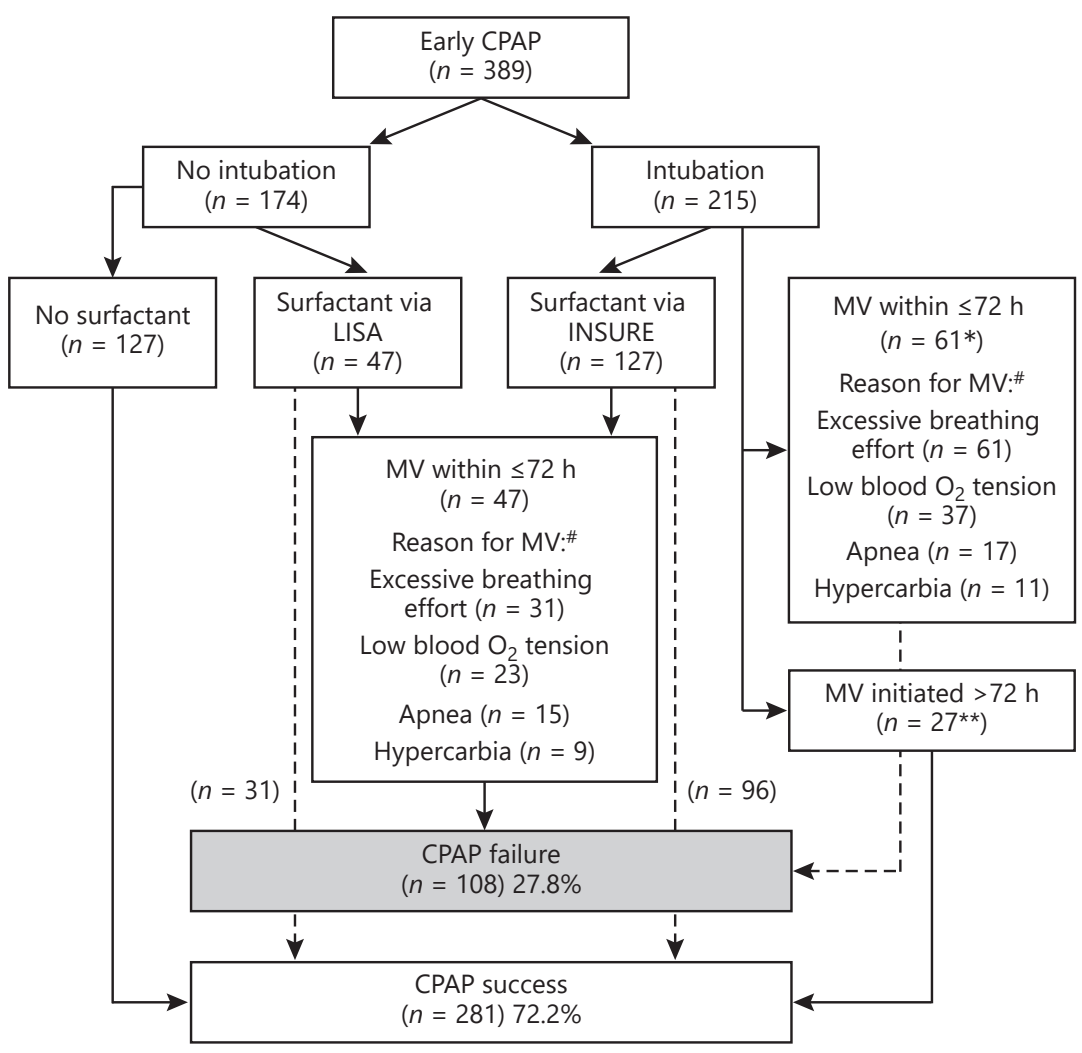

Birth weight was less predictive, with an AUC of 0.64 , 95\% CI 0.57-0.70, and a best discrimination threshold of $1,010 \mathrm{~g}$.

\section{CPAP Results and Complication Rate}

CPAP failure was associated with increased odds of unfavorable outcomes, including significantly higher rates of mortality (OR 26.5, 95\% CI 7.8-90.1), an approximately 2 - to 5 -fold increase in the incidence of typical complications of prematurity, and respiratory complications encompassing moderate to severe $\mathrm{BPD}$, as shown in Table 3.

\section{Discussion}

Fig. 2. ROC curve for prognosing CPAP failure according to highest $\mathrm{FiO}_{2}$ level in the second hour of life. The cutoff point corresponds to the highest specificity and sensitivity values.
The designation of risk factors for CPAP failure is crucial in the selection of an optimal method of respiratory support and may also help to find the best time point for exogenous surfactant administration. 
Table 2. Prediction of CPAP failure based on logistic regression models

\begin{tabular}{|c|c|c|c|c|c|c|}
\hline Predictor & OR (95\% CI) & $p$ & OR (95\% CI) & $p$ & OR $(95 \% \mathrm{CI})$ & $p$ \\
\hline Male gender & $1.05(0.67-1.64)$ & 0.821 & $1.00(0.61-1.66)$ & 0.986 & - & - \\
\hline Gestational age, weeks & $0.81(0.68-0.97)$ & 0.021 & $0.99(0.78-1.26)$ & 0.947 & - & - \\
\hline Antenatal steroids & $1.51(0.69-3.63)$ & 0.324 & $1.37(0.58-3.58)$ & 0.497 & - & - \\
\hline Cesarean delivery & $1.62(0.86-3.23)$ & 0.148 & $1.16(0.55-2.54)$ & 0.708 & - & - \\
\hline $\mathrm{FiO}_{2}-1$ st h of life & $1.04(1.02-1.06)$ & $<0.001$ & $1.02(1.00-1.04)$ & 0.072 & $1.02(1.00-1.04)$ & 0.061 \\
\hline $\mathrm{FiO}_{2}-2 \mathrm{nd}$ h of life & $1.08(1.05-1.10)$ & $<0.001$ & $1.06(1.04-1.09)$ & $<0.001$ & $1.06(1.04-1.09)$ & $<0.001$ \\
\hline Initial CPAP pressure, $\mathrm{cm} \mathrm{H}_{2} \mathrm{O}$ & $1.02(0.73-1.40)$ & 0.911 & $0.97(0.67-1.38)$ & 0.865 & - & - \\
\hline
\end{tabular}

Significant $p$ values are indicated in bold. CPAP, continuous positive airway pressure; $\mathrm{FiO}_{2}$, fraction of inspired oxygen.

Table 3. Clinical outcomes

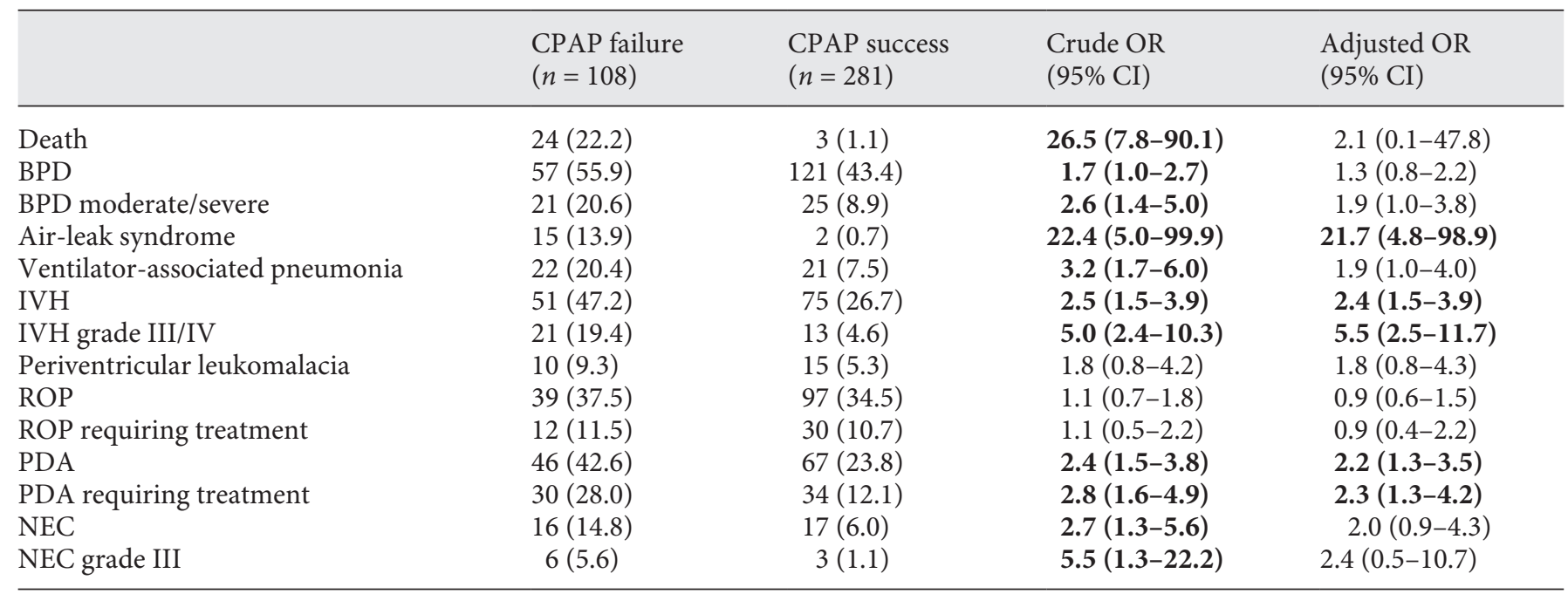

CPAP data are presented as $n$ (\%). Crude and adjusted ORs for adverse outcomes, comparing odds in the CPAP-failure group with those of the CPAP-success group. Adjusted ORs were derived from the multivariate logistic regression model, with the following covariates: gestational age, birth weight, and 5-min Apgar score. Significant ORs are indicated in bold. CPAP, continuous positive airway pressure; BPD, bronchopulmonary dysplasia; IVH, intraventricular hemorrhage; ROP; retinopathy of prematurity; PDA, persistent ductus arteriosus; NEC, necrotizing enterocolitis.

In our study, we prospectively followed a cohort of 389 newborns ( $<30$ gestational weeks) treated with early nasal CPAP. The overall rate of CPAP failure was $27.8 \%$. This confirms the high efficacy of early CPAP, which was similar to that reported in other studies with the same gestational age as our study population (failure rates: $20.6 \%-$ Rocha et al. [11]; 34\% - De Jaegere et al. [10]). However, a high success rate of early CPAP must be interpreted with some caution. A certain percentage of premature in- fants inevitably requires intubation in the delivery room. This study focused solely on those infants in whom an attempt of noninvasive ventilation was made. In Polish centers, such babies constitute a minority (about 38\%) of all infants born before 30 weeks based on the results of a national survey of 987 preterm newborns with RDS [13], where 333 of 573 (58\%) infants $<30$ weeks were intubated in the delivery room (data on file). 
While investigating the predictors of CPAP failure, a number of pre- and postnatal factors were analyzed. We found no statistically significant association for delivery method, multiple pregnancy, sex, or the use of antenatal steroids.

Stepwise logistic regression identified the final multivariate independent predictors for CPAP failure, which were $\mathrm{FiO}_{2}$ in the second hour of life and birth weight. Although the latter strongly correlates with gestational age, in the final regression model, birth weight and not gestational age played a role. In the model elaborated by De Jaegere et al. [10], the risk of CPAP failure was highest, with birth weight $\leq 800 \mathrm{~g}$. In our study, the threshold established in ROC analysis had a remarkably higher value $(1,010 \mathrm{~g})$, possibly reflecting the impact from multiple treatment centers, of which not all had equal experience. Additionally, disparities in prenatal care among the participating centers could have affected the results.

Of special interest was the $\mathrm{FiO}_{2}$ in the first two hours of life. Univariate analysis showed a significant association with CPAP failure, and each percentage point of oxygen required in the second hour of life increased the risk of unfavorable outcome by $7.5 \%$. Additionally, in the final multivariate regression model, $\mathrm{FiO}_{2}$ was the most potent prognostic factor of CPAP failure. This simple indicator of oxygenation, not requiring blood sampling or sophisticated equipment, warranted further exploration. In previous studies, $\mathrm{FiO}_{2}$ was analyzed as a dichotomous variable, and the risk for CPAP failure was compared between infants with $\mathrm{FiO}_{2}$ below and above the prespecified arbitrary thresholds $[7,11]$. In contrast to this approach, we used ROC curve analysis to determine the $\mathrm{FiO}_{2}$ cutoff level that best indicated CPAP failure. In our cohort, this $\mathrm{FiO}_{2}$ threshold was 0.29 . Only two studies, both of which were retrospective, evaluated the $\mathrm{ROC}$ curve for $\mathrm{FiO}_{2}$. De Jaegere et al. [10] reported an $\mathrm{FiO}_{2}$ threshold of 0.25 but did not provide any data on the accuracy and prognostic performance of this predictor. In turn, Dargaville et al. [5] reported only the approximate $\mathrm{FiO}_{2}$ threshold of "around 0.3 ," averaged for their two subsets of studied infants stratified by gestational age (25-28 and 29-32 weeks).

Despite achieving statistical significance, the ROC analysis findings must be interpreted considering the indices of the accuracy of the predictor. The extremely low $p$ value for the AUC $(p<0.0001)$ and the relatively narrow confidence interval correspond to the high precision with which the ROC curve was determined, and was, among others, a function of a large sample size. However, they are not the exponents of prognostic power. Our ROC curve for $\mathrm{FiO}_{2}$ had an AUC of 0.7, which indicated ade- quate but not great diagnostic performance, and was lower than that in the study by Dargaville et al. [5] (AUC = $0.8)$. The $\mathrm{FiO}_{2}$ threshold of 0.29 had good sensitivity (73\%) and only acceptable specificity (57\%), reflecting the clinically observed "overlap" of $\mathrm{FiO}_{2}$ distribution in CPAP-success and CPAP-failure populations, with some infants avoiding MV despite higher oxygen requirements. Therefore, $\mathrm{FiO}_{2}>0.29$ should be treated as a precautionary alert and an indicator of the need for surfactant administration to enhance the chance for CPAP success but not as an infallible predictor of CPAP failure.

In all study sites, the criteria for surfactant followed the European RDS guidelines [3]. $\mathrm{FiO}_{2}$ before surfactant therapy was higher in babies who failed CPAP; however, this was unrelated to the age at surfactant administration (Table 1), which was not significantly different. Additionally, the technique of administration (LISA vs. INSURE) did not affect the time of intervention.

In our study, we observed significantly higher odds of complications of prematurity in the CPAP failure group. Also, after risk adjustment, the ORs were markedly higher for severe morbidity, including grade III/IV IVH, air leaks, and PDA requiring treatment. These findings are in alignment with those of other papers, which also report a striking difference in the rates of complications in infants with CPAP failure $[5,6,14]$.

In previous studies, predictors of CPAP failure were investigated either with retrospective data $[5-7,10]$ or in a relatively small cohort of preterm infants $(n=131$, Rocha et al. [11]). For our analysis, we used a prospective collection of data from a large population of very premature newborns with predefined medical criteria. The results add to the previous body of work on the role of oxygen requirement in the first hours after birth in predicting CPAP outcome. The $\mathrm{FiO}_{2}$ threshold found in our study (0.29) might be considered along with similar thresholds of other authors (0.25 - De Jaegere et al. [10]; 0.3 - Dargaville et al. [5]) during the next revision of the European guidelines on the management of RDS. It may be reasonable to introduce a uniform $\mathrm{FiO}_{2}$ requirement $(>0.3)$ for surfactant therapy instead of the current two requirements ( $>0.3$ in infants $\leq 26$ weeks and $>0.4$ in those $>26$ weeks [3]). A single lower $\mathrm{FiO}_{2}$ threshold might be more closely followed in daily practice, which could potentially improve treatment outcomes.

One limitation of these analyses is the small number and limited representativeness of the most immature babies in the study cohort, with only 4 infants at 24 weeks gestation and 11 infants at 25 weeks gestation. Additionally, we lack comparative data on clinical outcomes in in- 
fants in whom nasal CPAP was started later or who were left on a trial of spontaneous breathing.

Given that the study involved multiple locations, the variability between sites is a major concern. We believe, however, that this does not impact the significance of our findings. The inclusion of neonate assignment (center factor) to the multivariate regression showed that it was irrelevant for the prediction of the CPAP outcome. The model that omitted the center factor had a significantly higher goodness of fit and better parsimony.

In conclusion, the efficacy of nasal CPAP in very premature newborns justifies its use as an initial method of respiratory support. The failure rate in infants $<30$ weeks gestation was $27.8 \%$, with the ultimate prognostic factors being birth weight and $\mathrm{FiO}_{2}$ exceeding 0.29 in the second hour of life. Nonresponders have a significant increase in the incidence of complications of prematurity.

\section{Acknowledgements}

The authors are grateful to all the investigators who collected data in this study (in alphabetical order):

St. Sophia's Specialist Hospital, Warsaw: Ewa Adamska; University Clinical Center, Gdańsk: Monika Bartosik-Woźniacka; Provincial Hospital, Bielsko-Biala: Katarzyna Bielak-Szcześniak, Ewelina Laszczak, Danuta Zimmer; Independent Public Specialized Health Care Center “Zdroje," Szczecin: Marzena Blachowska; Ujastek Medical Center, Krakow: Jadwiga Bublik, Olga Gadzielińska, Marek Jaszczak; Jan Paweł Lauterbach, Elżbieta RafińskaWażny, Renata Saganowska; Provincial Integrated Hospital, Elbląg: Barbara Chomik; Centre for Gynaecology, Obstetrics and Neonatology, Opole: Dominika Chrzanowska-Grzywacz; University Women's and Newborn Health Center, Warsaw: Agnieszka Drozdowska-Szymczak, Anna Rybakiewicz-Kamińska, Ewa Wilkos; Provincial Specialist Hospital, Wroclaw: Elżbieta JasiakPałczyńska; M. Pirogow Provincial Specialist Hospital, Lodz: Marcin Kęsiak; Jan Biziel University Hospital No. 2, Bydgoszcz: Piotr Korbal, Iwona Sadowska-Krawczenko; Professor Witold Orłowski Independent Public Clinical Hospital, Warszawa: Maria Kostro, Maria Wilińska; Independent Public Clinical Hospital No. 4, Lublin: Marzena Kostuch; COPERNICUS Health Center Ltd., Gdansk: Iwona Kuehnel; University Clinical Hospital, Bialystok: Elżbieta Kulikowska; Bielany Hospital, Warsaw: Magdalena Kurzątkowska; University Clinical Hospital, Wroclaw: Dorota LisowskaMikołajków; Independent Public Clinical Hospital No. 2, Szczecin: Beata Łoniewska; Department of Newborns' Infectious Diseases, Poznan University of Medical Sciences, Poznan: Katarzyna Mielnik; Department of Neonatology, Poznan University of Medical Sciences, Poznan: Natalia Neumann-Klimasińska; University Hospital, Krakow: Patrycja Ochońska, Dorota Pawlik, Renata Radziszewska; Provincial Specialist Hospital, Olsztyn: Łukasz Osiński; Polish Mother's Memorial Hospital Research Institute, Lodz: Barbara Pawłowska; Institute of Mother and Child, Warszawa: Marzanna Reśko-Zachara; Masovian Bródno Hospital, Warsaw: Paweł Ruszczykowski, Marta Tomaszewska; Princess Anna
Mazowiecka Clinical Hospital, Warsaw: Anna Sonczyk-Zapała; Specialist Hospital No. 2, Bytom: Katarzyna Szczepańska; St. John of God Independent Public Provincial Hospital, Lublin: Elżbieta Szmit; Provincial Specialist Hospital, Czestochowa: Grzegorz Zieliński.

\section{Statement of Ethics}

The study protocol was approved by the Ethics Committee of Warsaw Medical University (ref. No. AKBE/154/16), in accordance with the principal investigator's affiliation. This paper presents the results of an observational study only collecting routinely available data from the hospital records and did not involve any direct intervention to participants. All parents/legal guardians gave written consent for all diagnostic and therapeutic procedures, in accordance with local law and practices. No sensitive or patientidentifiable data were collected in this project.

\section{Disclosure Statement}

R.H. is employed by Chiesi Poland, the sponsor of the study. E.G., M.R., and M.K.B.-K. received honoraria from Chiesi Poland for scientific presentations and participation on advisory boards. T.S. received honoraria for lectures from Chiesi Poland.

\section{Funding Sources}

The study was financially supported by Chiesi Poland Sp. z o.o., a subsidiary of Chiesi Farmaceutici, Italy.

\section{Author Contributions}

R.H., M.K.B.-K., E.G., and M.R. were involved in planning and supervision of the study. E.G., T.S., and R.H. drafted the manuscript with input from all authors. R.H. designed the figures. M.R. compiled the literature sources and provided critical feedback. All authors discussed the results and approved the version to be published.

\section{References}

1 Parat S, Mhanna MJ. Respiratory management of extremely low birth weight infants: survey of neonatal specialists. World J Pediatr. 2016 Aug;12(3):314-9.

2 Trevisanuto D, Satariano I, Doglioni N, Criscoli G, Cavallin F, Gizzi C, et al.; Neonatal Resuscitation Study Group, Italian Society of Neonatology. Changes over time in delivery room management of extremely low birth weight infants in Italy. Resuscitation. 2014 Aug;85(8):1072-6.

3 Sweet DG, Carnielli V, Greisen G, Hallman M, Ozek E, Plavka R, et al. European Consensus Guidelines on the Management of Respiratory Distress Syndrome - 2016 Update. Neonatology. 2017;111(2):107-25. 
4 Subramaniam P, Ho JJ, Davis PG. Prophylactic nasal continuous positive airway pressure for preventing morbidity and mortality in very preterm infants. Cochrane Database Syst Rev. 2016 Jun;6:CD001243.

5 Dargaville PA, Aiyappan A, De Paoli AG, Dalton RG, Kuschel CA, Kamlin CO, et al. Continuous positive airway pressure failure in preterm infants: incidence, predictors and consequences. Neonatology. 2013;104(1):814.

6 Dargaville PA, Gerber A, Johansson S, De Paoli AG, Kamlin CO, Orsini F, et al.; Australian and New Zealand Neonatal Network. Incidence and Outcome of CPAP Failure in Preterm Infants. Pediatrics. 2016 Jul;138(1): e20153985.
7 Fuchs H, Lindner W, Leiprecht A, Mendler MR, Hummler HD. Predictors of early nasal CPAP failure and effects of various intubation criteria on the rate of mechanical ventilation in preterm infants of $[\{L T\}] 29$ weeks gestational age. Arch Dis Child Fetal Neonatal Ed. 2011 Sep;96(5):F343-7.

8 Dimitriou G, Fouzas S, Giannakopoulos I, Papadopoulos VG, Decavalas G, Mantagos S. Prediction of respiratory failure in late-preterm infants with respiratory distress at birth. Eur J Pediatr. 2011 Jan;170(1):45-50.

9 Siew ML, van Vonderen JJ, Hooper SB, et al. Very preterm infants failing CPAP show signs of fatigue immediately after birth. PLoS One. 2015 Jun;10(6):e0129592.

10 De Jaegere AP, van der Lee JH, Canté C, van Kaam AH. Early prediction of nasal continuous positive airway pressure failure in preterm infants less than 30 weeks gestation. Acta Paediatr. 2012 Apr;101(4):374-9.
11 Rocha G, Flôr-de-Lima F, Proença E, Carvalho C, Quintas C, Martins T, et al. Failure of early nasal continuous positive airway pressure in preterm infants of 26 to 30 weeks gestation. J Perinatol. 2013 Apr;33(4):297-301.

12 Borszewska-Kornacka MK, Dobrzanska A, Gulczynska E, et al. Standardy opieki medycznej nad noworodkiem w Polsce [Standards of medical care for a newborn in Poland] Warsaw: Media Press; 2015.

13 Borszewska-Kornacka MK, Kostuch M, Korbal P, Krajewski P; Polish RDS Study Group. Strategies of using surfactant: results of the first Polish national survey of daily practice. Dev Period Med. 2015 Jul-Sep;19(3 Pt 1): 271-6.

14 Ammari A, Suri M, Milisavljevic V, Sahni R, Bateman D, Sanocka U, et al. Variables associated with the early failure of nasal CPAP in very low birth weight infants. J Pediatr. 2005 Sep;147(3):341-7. 